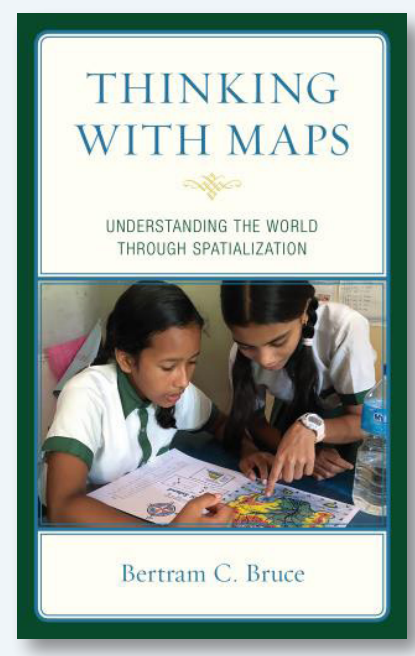

\title{
THINKING WITH MAPS: UNDERSTANDING THE WORLD THROUGH SPATIALIZATION
}

By Bertram C. Bruce

Roman \& Littlefield, 2021

174 pages

Softcover: \$40, ISBN 978-1-4758-5929-4

Review by: Richard Bohannon (he/him), Metropolitan State University

Bertram Bruce is a MULTidisciplinARY scholar with a background in computer science, whose work has more recently focused especially on teaching and learning. As its title implies, maps are the primary concern of his new book, Thinking with Maps: Understanding the World Through Spatialization, which looks at education and the role of maps in how we learn about the world, both inside and outside of the classroom. This book is not written with cartographers and geographers as the primary audience, but for a broader audience less familiar with maps.

In the Introduction, Bruce outlines a basic and useful insight that he repeats in various ways throughout the volume: humans have developed three systems of symbols to understand the world and communicate with each other. Written language comprises the first, and mathematics the second. Modern educational systems revolve around these two symbol systems, and for good and obvious reasons; reading, writing, and arithmetic are foundational skills.

But Bruce argues that a third system of symbols-spatialization-has become increasingly essential "as the world becomes more interconnected, and as our understanding of ecological, political, and cultural interconnections grows" (xxvii). The use of the term "spatialization" here is apt, for while Bruce does write about traditional maps throughout the book, he means something broader-including not just geographic maps but concept maps, charts, histograms, and the like. His use of the term "map" is intentionally expansive and meant to cover this wide array of visualizations. While this would, arguably, be too broad of a definition for some cartographers, it's an understandable rubric given the book's aim. The author wants us to consider spatialization as a way of understanding the world, on par with both writing and math. Maps can be used not just to learn the basics of geography or for memorizing the names and location of countries, cities, and rivers, but to understand spatial relationships more broadly, perform basic spatial analysis, and understand how things connect to each other.

Bruce structures the book around different maps uses, ending with a reflection on what counts as a map. The first chapter starts with the basics: using maps to navigate and find directions. Much of the chapter is very simple-several pages are spent explaining how road and trail maps are used, for example-before arguing that maps help us understand where we are not only practically, but existentially, as "the means we employ to make sense of our life in context” (19).

Chapter Two further explores the ways that maps construct the world around us, with the role of maps in drawing political boundaries serving as a clear example. Most of the chapter revolves around the city of Paris, and especially the history of street names across different neighborhoods and arrondissements, before moving to hermeneutics and the way maps are always up for interpretation and consequently interpreted differently from different perspectives. While Bruce shows a detailed knowledge of Parisian street names, his broad understanding of what counts as a map - here the city itself is construed "as a map of Parisian history" (37) - misses an opportunity to draw on the work 
of the many geographers (such as Denis Wood or John Pickles) who have written on similar themes.

While the third chapter is titled "Learning," it dwells more on role of maps (again, broadly construed) and science. "Maps can make data come alive," Bruce writes. "One's capacity to do science is defined, to a large extent, by the ability to read and create maps and to move between visual and conceptual modes of understanding" (44). Most of the chapter is filled with examples of how maps can be used to explore and make sense of data, but the more interesting contribution comes toward the end, where he provides several examples of how maps can be used in educational settings to promote learning. At a grade school in Nepal, for instance, students use maps to explore their local environment and culture as well as to record what they have learned, and children at a school in Tucson, Arizona, use maps of real and imaginary gardens to help learn geometry.

This educational theme continues through subsequent chapters. Chapter Four is a brief exploration of maps as artistic expression, as a way for people to learn by being "actively engaged in making meaning for themselves" (80). Once again, the idea of a "map" here is often quite expansive-including, for example, several pages on musical scores and notation, or "maps of music" (76). The focus is less on maps themselves, and more on how the artistic use and creation of maps "can be a major factor in enlarging civic intelligence and promoting democratic society" (80).

Chapter Five begins with three nineteenth-century examples, well known to many cartographers, that are used to briefly describe how maps can be used to solve problems: John Snow's cholera maps, Charles Booth's poverty maps, and Florence Kelley's Hull-House maps. These early thematic maps exemplify one of the core educational advantages of modern GIS data, which allows mapmakers to discover connections between different data layers (of poverty levels, for example, or public health data). In making such connections, Bruce argues that maps become "performative utterances," a term borrowed from the philosophy of language that refers to words "that change social reality rather than describe a pre-existing reality" (91). He points out that maps such as those by Snow, Booth, and Kelley not only describe a place, but change how their audiences perceive that place; they create a new sense of place.

The sixth and seventh chapters pick up on this theme, providing examples of learning through the mapmaking process. In a discussion of a map exercise for students learning about early US history, for instance, Bruce writes that "the idea is to encourage students to use map reading and mapmaking as tools for thinking. Rather than memorizing dates for significant events, they make visual and tangible the story they are learning" (104). This is the heart of Bruce's argument: that maps and spatial thinking are critical across the school curriculum, beyond their more obvious role in learning geography.

The eighth and final chapter revisits the question of how we define the term "map" through an evolving parade of quite diverse examples, ranging from cladograms (phylogenetic charts demonstrating the relationships between groups of organisms) to the stick charts traditionally used for ocean navigation in the Marshall Islands. Bruce ultimately argues a map is "a story the author expresses or the reader infers, built upon a spatialized representation of the relations among elements of some system" (159). Again, this definition encompasses a wide range of visualizations; while it can lead to some interesting examples, it also dilutes the distinctiveness of the more traditional, geographic map.

Many readers of this journal might wish Bruce had expanded more on the book's core ideas about education, and spent less time providing introductory overviews of maps and other visualizations. While he does offer several examples of students learning through making maps, they are always brief, and he quickly moves along to another topic that often seems unrelated. A more systematic exploration of the pedagogical possibilities and limitations of mapping outside of the geography classroom would have been enlightening.

Thinking with Maps also stems from the author's broader interest in "democratic education," or an education guided by values and ethics. Especially given the historic (and contemporary) role of maps and GIS in colonization and ecologically destructive industries, which Bruce himself discusses briefly in several chapters, a more thorough and rigorous discussion of how maps can inform a democratic education would be welcome. Nonetheless, this book leaves cartographers, professional or otherwise, with an important question: in what ways do our maps help or hinder the development of "an enlarged capacity to critique, participate in, and sustain a flourishing society," (168) for which Bruce calls? 